\title{
CORRESPONDENCE
}

\section{Hematogone hyperplasia - A double edged sword}

\author{
Aditi Kundoo $^{1} \quad$ Sabina Langer $^{*} \quad$ Divij Sachdeva $^{2} \quad$ Anupam Sachdeva $^{2} \quad$ Jyoti Kotwal $^{1}$
}

Hematogones are lymphoid progenitor cells usually comprising $<1 \%$ of marrow nucleated cells. Hematogone hyperplasia is defined as $>5 \%$ hematogones in bone marrow ${ }^{[1]}$.

Hematogone hyperplasia is seen in many reactive and neoplastic conditions such as autoimmune cytopenia(s), post viral infections, Hodgkin/ Non Hodgkin lymphoma, acute myeloid leukaemia, post chemotherapy or stem cell transplant bone marrow ${ }^{[2]}$. However, occasionally a marked reactive process like hematogone hyperplasia can mask an important underlying morphology. It is further compounded in cases where the diagnostic cells are few in number. Clinching a diagnosis in such cases becomes increasingly difficult.

We here cite a case where hematogone hyperplasia masked an important diagnosis of acute megakaryoblastic leukaemia. This case also highlights the importance of use of multimodalities to diagnose a haematological disorder.

A two-year old girl presented to paediatric out patient department(OPD)with history of bruises over lower leg since 15 days and thrombocytopenia $\left(63 \times 10^{9} / \mathrm{L}\right)$. She was being treated for suspected dengue by a non allopathic medicine (Tinospora Cordifolia) known to have immunomodulator and antineoplastic effect. The complete blood count was hemoglobin $(\mathrm{Hb}) 86 \mathrm{~g} / \mathrm{L}$, platelet count $16 \times 10^{9} / \mathrm{L}$. Immature platelet fraction (IPF)- $4.5 \%$ ) and total leukocyte count (TLC) $7.8 \times 10^{9} / \mathrm{L}$. Abnormal blastoid cells $(2 \%)$ were reported on peripheral blood smear and bone marrow studies were advised. Bone mar-

\footnotetext{
Received: September 24, 2020 Accepted: October 20, 2020 Revised: October 28, 2020 Published: November 6, 2020

* Correspondence to: Sabina Langer, MBBS, MD Pathology, Senior Consultant, DepartCorrespondence to: Sabina Langer, MBBS, MD Pathology, Senior Consultant, Depart-
ment of Haematology, Sir Ganga Ram Hospital, Rajinder Nagar, New Delhi 110060, India; Email: sabinapost@yahoo.com

${ }^{1}$ Department of Haematology, Sir Ganga Ram Hospital, Rajinder Nagar, New Delhi 110060 , India

2 Department of Paediatric Hematoncology, Sir Ganga Ram Hospital, Rajinder Nagar, New Delhi 110060, India

Citation: Kundoo A, Langer S, Sachdeva D et al. Hematogone hyperplasia - A double edged sword. Curr Cancer Rep, 2021, 3(1): 64-67.

Copyright: $\odot 2020$ Sabina Langer, et al. This is an open access article distributed under the terms of the Creative Commons Attribution License, which permits unrestricted use, distribution, and reproduction in any medium, provided the original author and source are credited.
}

row aspirates received were grossly haemodilute; imprints were also moderately to sparsely cellular. There was presence of atypical cells with fine homogenous chromatin, inconspicuous nucleoli \& scant cytoplasm. These constituted $19 \%$ of the total nucleated cells. Erythropoiesis was normoblastic with marked paucity of megakaryocytes. In flowcytometric immunophenotypic analysis, hematogone hyperplasia was seen constituting $25 \%$ of all acquired cells after excluding doublets and debris (Figure 1). These were recognized by their CD38 and CD19 positivity with sequential maturation pattern visible on CD10 vs. CD20 and CD10 vs. CD38 plots. Blasts expressing CD7 and CD13/33 were also identified and comprised $2.5 \%$ of the total events. Surprisingly, bone marrow biopsy revealed MF grade 2 to 3 fibrosis, megakaryocytic hyperplasia with abnormal megakaryocytes, micromegakaryocytes and megakaryoblasts. Immunohistochemistry of CD61 highlighted megakaryocytes and megakaryoblasts (Figure 2(A) and (B)). Retrospectively bone marrow aspirate slides revealed occasional blasts with cytoplasmic blebbing. A diagnosis of acute megakaryoblastic leukaemia (AML-M7) was considered. A repeat bone marrow study was advised after the effects of the medicine had weaned off.

Repeat bone marrow biopsy was done 20 days later with $\mathrm{Hb} 125 \mathrm{~g} / \mathrm{L}$, platelet count $39 \times 10^{9} /$ Land TLC 12 $\times 10^{9} / \mathrm{L}$ There were $28 \%$ circulating blasts in the peripheral blood. Bone marrow aspirates were poorly cellular with an increase in blasts comprising $30 \%$ of the total marrow nucleated cells. Blasts were intermediate to large in size with high nuclear to cytoplasmic ratio, round to oval regular nuclei, fine chromatin, 1-2 prominent nucleoli and appreciable amount of pale basophilic cytoplasm with cytoplasmic blebbing. They were negative for myeloperoxidase stain (Figure 2(C) and (D)). Accompanying hematopoietic cells were myeloid, erythroid precursors and lymphocytes. Flowcytometric analysis of the aspirate revealed $24.0 \%$ population in the CD45 dim blast region (Figure 3 ). These blasts were cytoplasmic MPO negative with CD33, CD117 and cytoplasmic CD41a positivity confirming them to be megakaryoblasts. 

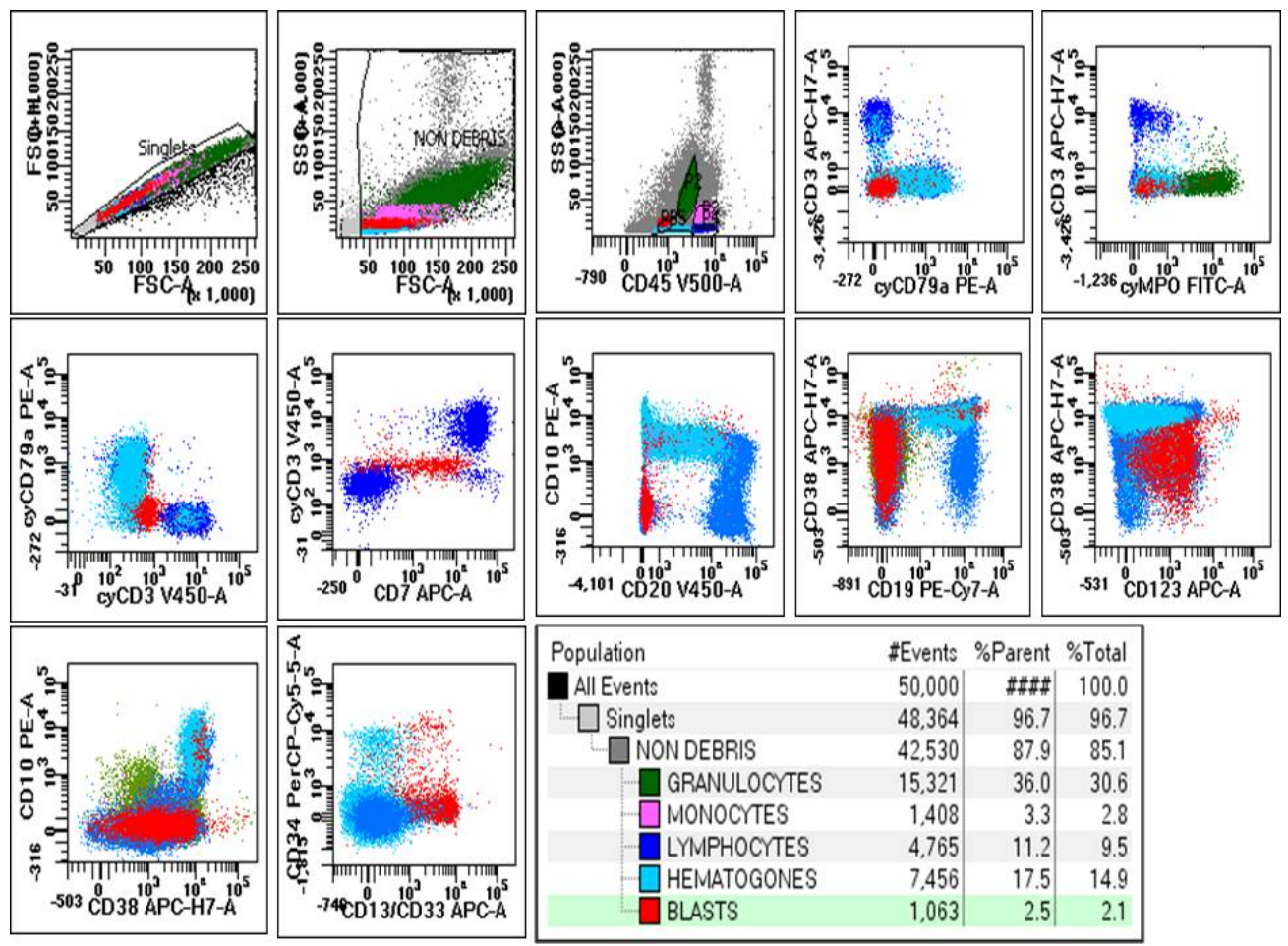

Figure 1. Flowcytometric dot plots at initial presentation with hematogone hyperplasia with $2.5 \%$ blasts. Hematogones were CD10, CD20, CD38, CD19 and CD34 positive and show sequential maturation pattern. Blasts were CD7 and CD13/CD33 positive

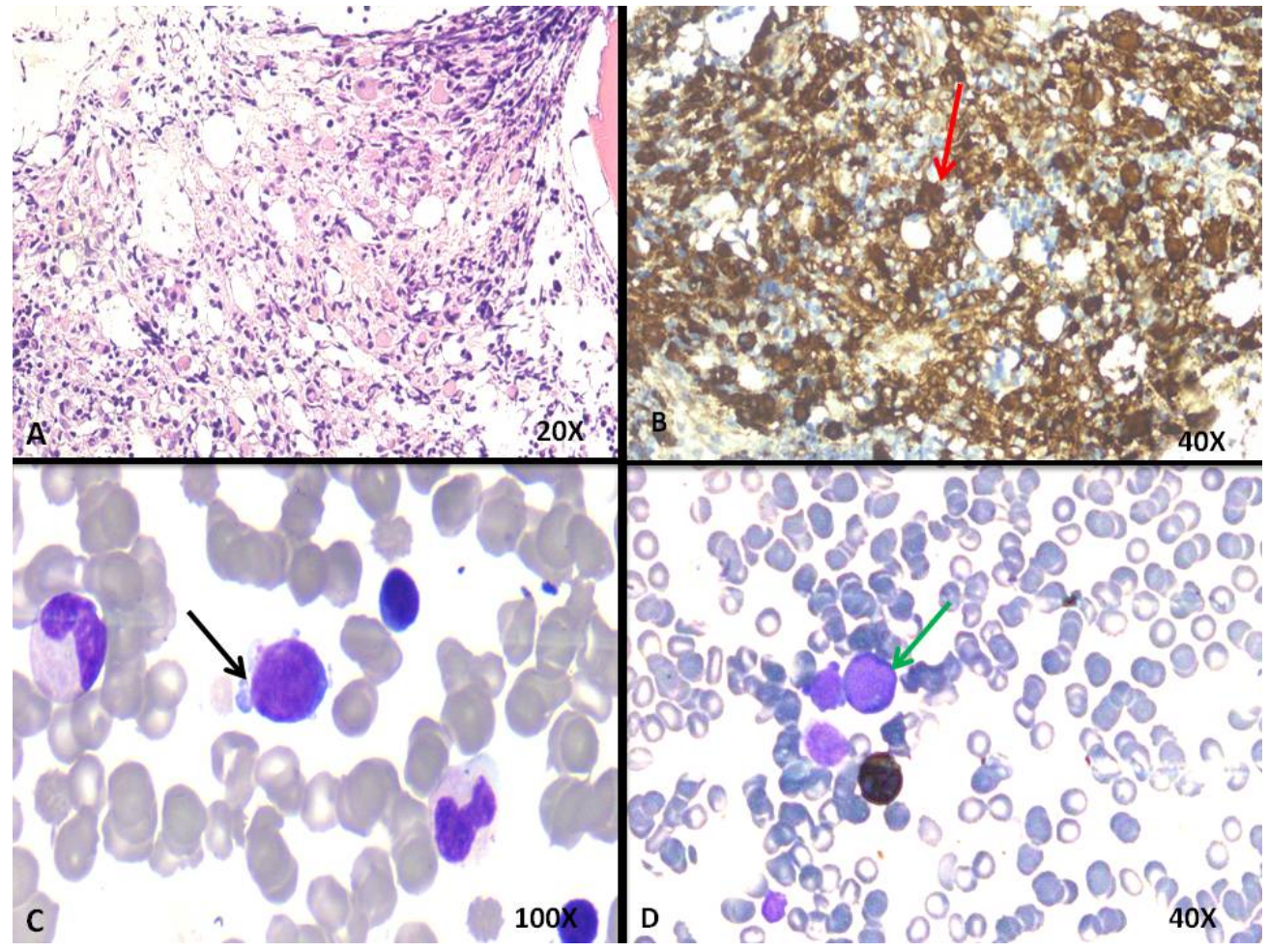

Figure 2. Histomicrographs showing (A) Bone marrow biopsy at the initial presentation with megakaryocytic hyperplasia, abnormal megakaryocytes, micromegakaryocytes and megakaryoblasts along with marrow fibrosis; (B) Immunohistochemistry for CD61 highlighted megakaryoblasts and megakaryocytes; (C) Repeat bone marrow aspirates with megakaryoblasts (intermediate to large in size with high nuclear to cytoplasmic ratio, round to oval regular nuclei, fine chromatin, 1-2 prominent nucleoli and appreciable amount of pale basophilic cytoplasm with cytoplasmic blebbing); (D) Myeloperoxidase negative blasts 


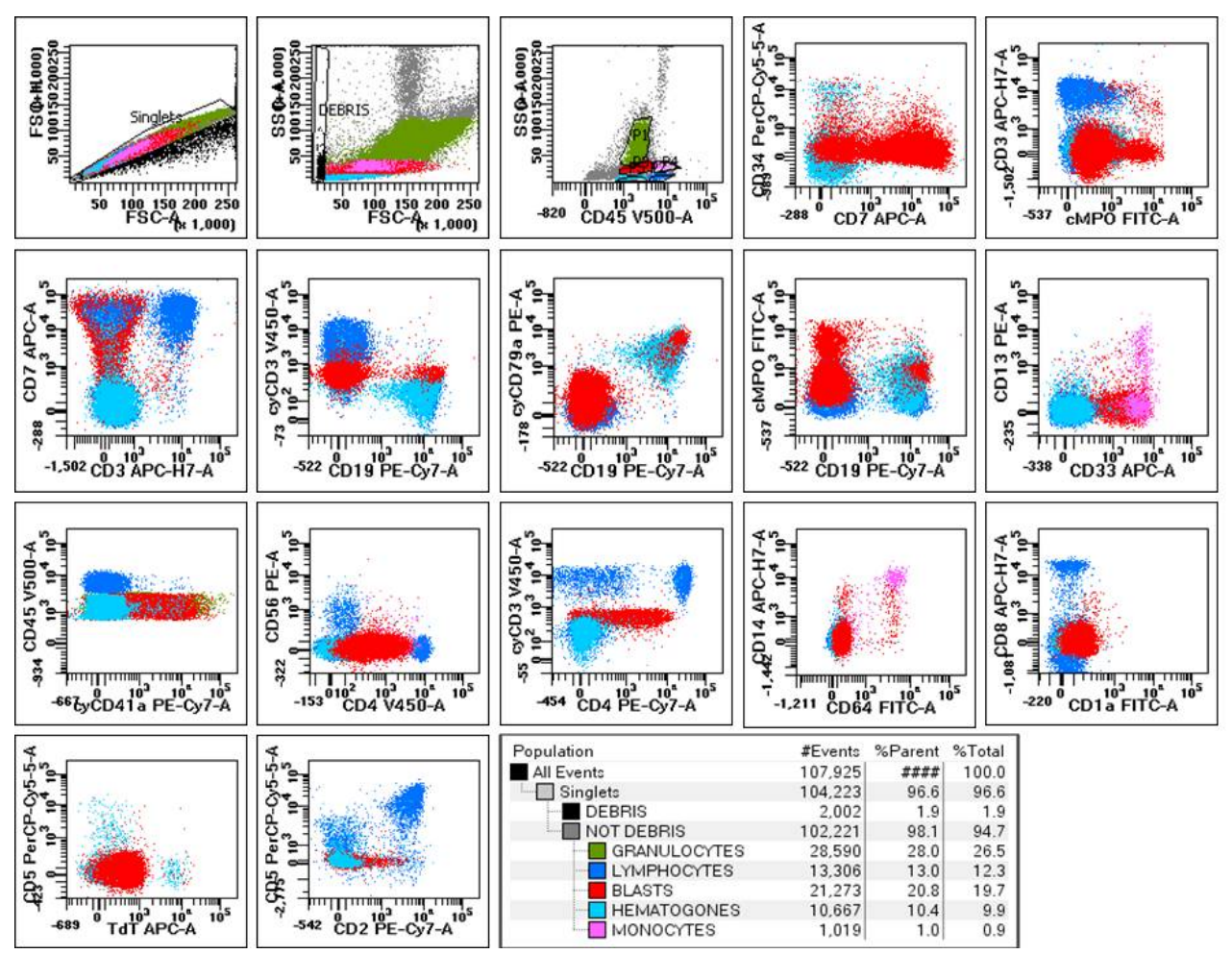

Figure 3. Flowcytometric dot plots of repeat aspirate with $24 \%$ blasts. These blasts were CD34, CD117, cytoplasmic CD41, CD71, CD33, HLA-DR, CD123, CD4, CD7 positive

These had aberrant CD7 and CD 4 expression. These were positive for CD34, CD71, HLA-DR and CD123 and negative for $\mathrm{B}$ lineage (CD79a and CD19); $\mathrm{T}$ lineage (cytoplasmic and surface CD3, CD2, CD8, and CD5) and monocytic (CD11b, CD56, CD14 and CD64) markers. In addition, there were $10.8 \%$ hematogones. The final diagnosis of acute megakaryoblastic leukaemia with aberrant CD7 and CD4 expression was rendered. Cytogenetics and molecular studies reported normal karyotype. She was given 4 cycles of chemotherapy according to COG protocol. Bone marrow studies done 3 months later showed morphological remission with $0.1 \%$ minimal residual disease. However subsequently the child went in relapse and succumbed to disease.

Hematogones even though is a marrow regenerative response, can camouflage the underlying pathology especially such as in megakaryoblastic leukaemia where due to marrow fibrosis diagnostic blasts are sparsely present in the peripheral blood and bone marrow. In addition, because of intake of unregulated medicines, presence of hematogone hyperplasia creates a further confusion of lymphoblasts creating a diagnostic dilemma.

A comprehensive analysis of literature was done from 1994-2020 and three authors ${ }^{[3-5]}$ have reported similar hindrance in the diagnosis of acute megakaryoblastic leukaemia because of presence of hematogones. AntonHarisi et al. ${ }^{[4]}$ reported 2 cases where there was either preferential uptake of hematogones owing to marrow fibrosis or there was dry tap and flowcytometry was repeatedly inconclusive. Repeated bone marrow aspirations were done to arrive at final diagnosis delaying the treatment. In a case study by Chaudhari et al. ${ }^{[5]}$ diagnosis of pre-B ALL was mistakenly made on flowcytometry when patient was not responding to chemotherapy, repeat aspirate and biopsy were done. On immunohistochemistry the blasts were positive for CD61, factor VIII, CD117 and HLADR and final diagnosis of acute megakaryocytic leukaemia was reported.

In the era of multiparametric flowcytometry many centres perform bone marrow biopsy only in cases of dry tap or diagnostic uncertainty ${ }^{[6]}$. However, this case reemphasises the importance of biopsy which gives a complete picture in context to number, morphology and distribution of cells. Though flowcytometry can pickup and analyse markers on small number of cells but bone marrow biopsy together with immunohistochemistry can supplement and give confidence in making a final diagnosis. Bone marrow regenerative changes are commonly associated with underlying haematological malignancy and these can divert our attention from the main pathology. Identification and employment of templates for all normal hematopoietic elements of bone marrow including mast cells, plasmacytoid dendritic cells, basophils, hematogones and normal myeloid progenitors can help 
in identification of abnormal cells with unfamiliar phenotype and differentiate them from the normal elements present in response to marrow regeneration. Employing this strategy will improve diagnostic accuracy and prevent misinterpretation of normal hemopoietic elements which are increased in reactive or regenerative bone marrows.

Take home message from this case is to employ larger panel of antibody markers when the suspicion of malignancy is high and the cellularity of sample is low. Correlation of flowcytometric findings with morphology especially bone marrow biopsy and immunohistochemistry is required. Awareness of such infrequent presentations will help us in scrutinizing cases extensively and prevent fallacies or delay in diagnosis.

\section{Acknowledgements}

The authors' contributions to this work are as follows: Dr. Aditi Kundoo drafted manuscript; Dr. Sabina Langer and Dr. Jyoti Kotwal worked up and diagnosed the case; Dr. Divij Sachdeva and Dr. Anupam Sachdeva treating physician.

\section{Conflict of interest}

There is no conflict of interest.

\section{References}

[1] McKenna RW, Washington LT, Aquino DB, et al. Immunophenotypic analysis of hematogones (B-lymphocyte precursors) in 662 consecutive bone marrow specimens by 4-color flow cytometry. Blood, 2001, 98(8): 2498-2507. https://doi.org/10.1182/blood.V98.8.2498

[2] Chantepie SP, Cornet E, Salaün V, et al. Hematogones: an overview. Leukemia Research, 2013, 37(11): 1404-1411. https://doi.org/10.1016/j.leukres.2013.07.024

[3] Richard G, Brody J and Sun T. A case of acute megakaryocytic leukemia with hematogones. Leukemia, 1993, 7(11): 1900-1903.

[4] Anton-Harisi M, Douna V, Baka M, et al. Acute megakaryoblastic leukemia with increased hematogones in children. Journal of Pediatric Hematology/Oncology, 2012, 34(8): e337-e340. https://doi.org/10.1097/MPH.0b013e3182678ddb

[5] Chaudhari J, Borges A, Bansal S, et al. AML M7 Misdiagnosed as ALL. Indian Journal of Hematology and Blood Transfusion, 2014, 30(Suppl 1): 199-201. https://doi.org/10.1007/s12288-013-0326-4

[6] Rack KA, van den Berg E, Haferlach C, et al. European recommendations and quality assurance for cytogenomic analysis of haematological neoplasms. Leukemia, 2019, 33(8): 1851-1867. https://doi.org/10.1038/s41375-019-0378 